\title{
EFECTO DE LA SUPLEMENTACIÓN SOBRE LA GANANCIA DE PESO Y RENDIMIENTO EN CANAL DE CORDEROS SUDAN
}

\section{EFFECT OF SUPPLEMENTATION ON WEIGHT GAIN AND CARCASS DRESSING OF SUDAN LAMBS}

\author{
Libardo Maza Angulo ${ }^{1}$, Moris Bustamante Yanez ${ }^{2}$, Juan Carlos Simanca Sotelo ${ }^{1 *}$, Mauricio Ruiz Ortiz ${ }^{4}$, \\ Guido Montaño Flórez ${ }^{5}$, Oscar Vergara Garay ${ }^{6}$
}

${ }^{1}$ MVZ, MSc. Universidad de Córdoba, Facultad de Medicina Veterinaria y Zootecnia, Departamento de Ciencias Pecuarias, Montería, e-mail: libardomaza@gmail.com; ${ }^{2}$ MVZ, Esp. Universidad de Córdoba, Facultad de Medicina Veterinaria y Zootecnia, Departamento de Ciencias Pecuarias, Montería, Córdoba, Colombia, e-mail: morisbustamante@correo.unicordoba.edu.co; ${ }^{3}$ MVZ, Esp. Universidad de Córdoba, Facultad de Medicina Veterinaria y Zootecnia, Departamento de Ciencias Pecuarias, Montería, Córdoba, Colombia, *autor para correspondencia, e-mail: j.c.simanca@hotmail.com; ${ }^{4}$ MVZ. Ejercicio particular, e-mail: mjrui_16@hotmail.com; ${ }^{5}$ MVZ. Ejercicio particular, e-mail: coratlans@hotmail.com; ${ }^{6}$ Zootecnista, PhD. Universidad de Córdoba, Facultad de Medicina Veterinaria y Zootecnia, Departamento de Ciencias Pecuarias, Montería, Córdoba, Colombia, e-mail: overgara@correo.unicordoba.edu.co

Rev. U.D.C.A Act. \& Div. Cient. 18/1): 283-286, Enero-Julio, 2015

\section{INTRODUCCIÓN}

La producción de ovinos (Ovis aries) ha sido tradicionalmente considerada por los campesinos de la costa norte de Colombia, como una actividad marginal de producción de proteína animal, para autoconsumo. Este tipo de explotación presenta bajos parámetros productivos, debido a las pocas investigaciones realizadas (MADR, 2006).

Los ovinos tienen un gran potencial de producción, debido a su capacidad de ingerir alimentos de bajo valor nutricional y transformarlos en proteína de alto valor biológico y la alta fertilidad, que se manifiesta en su madurez sexual precoz, la duración de su actividad sexual y los partos gemelares (Izaguirre et al. 2011); sin embargo, su potencial de producción en condiciones de pastoreo está afectado, principalmente, por la disminución de la oferta y la baja calidad del forraje, consumido durante la época seca. El objetivo de esta investigación es evaluar el efecto de la suplementación energético-proteica, sobre la ganancia de peso diaria posdestete y rendimiento de la canal en corderos de raza Sudan, manejados en condiciones de pastoreo.

\section{MATERIALES Y MÉTODOS}

Sitio de estudio y geoclima. El estudio, se realizó en la Granja experimental de la Facultad de Medicina Veterinaria y Zootecnia, de la Universidad de Córdoba, localizada en el corregimiento de Berastegui, Municipio de Ciénaga de Oro, departamento de Córdoba, Colombia. La zona, se encuentra clasificada como bosque húmedo tropical, ubicado a $8^{\circ 52}$ latitud norte y $75^{\circ} 54^{\prime}$ de longitud al oeste, altura a $18 \mathrm{msnm}$, temperatura promedio de $27^{\circ} \mathrm{C}$, humedad relativa de $85 \%$ y precipitación anual de $1.200 \mathrm{~mm}$.

Unidades experimentales y tratamientos. Se utilizaron siete corderos machos de raza criolla Sudan, de 6 meses de edad y $23,6 \pm 3,7 \mathrm{~kg}$ de peso promedio. Después del destete, los animales fueron distribuidos al azar, en dos grupos de tratamientos: T0: tres corderos no suplementados y T1: cuatro corderos suplementados.

Manejo de los animales y suplementación. Los animales del grupo T0 fueron manejados en pastoreo rotacional durante las horas del día, comprendidas entre las 8:00 a.m. y 4:00 p.m., en praderas de Angleton (Dychantium aristatum), con suministro permanente de agua; el pasto tuvo composición química del $25 \%$ de materia seca; 9,98\%, de proteína cruda; $58,43 \%$, de fibra detergente neutra (FDN); $50,74 \%$, de fibra detergente ácida (FDA) y 15,74\%, de ceniza; este análisis, se realizó en el laboratorio de nutrición animal de la Universidad de Córdoba (ICONTEC, 1999; ICONTEC, 2006; AOAC, 2002). Los animales del grupo T1 fueron manejados en pastoreo rotacional durante las horas del día, comprendidas entre las 8:00 a.m. y 4:00 p.m., en praderas de Angleton (Dychantium aristatum), con suministro permanente de agua y suplementados con $250 \mathrm{gr} / \mathrm{dí} / \mathrm{animal}$ de una mezcla de alimento a base de maíz molido, (50\%); salvado de arroz, (27\%); torta de soya, (20\%); sal mineralizada, (3\%), que aporto $17, \%$ de proteína cruda, 13,5\% de FDN, 7,8\% de FDA 
y, 7,5\% de ceniza. Este análisis, se realizó en el laboratorio de nutrición animal de la Universidad de Córdoba (ICONTEC, 1999; ICONTEC, 2006; AOAC, 2002).

Ganancia de peso. Para determinar la ganancia diaria de peso (GDP) de los animales, se hicieron pesajes cada 14 días, a las 7:00 a.m., durante 126 días. Previo a esto, los animales fueron sometidos a un periodo de acostumbramiento de 14 días; para realizar el pesaje, se empleó una báscula electrónica modelo PB2015 (D=100g).

Rendimiento de la canal. La edad aproximada al sacrificio fue de 10,5 meses, edad a la que los productores realizan el sacrifico de los animales. Para evaluar el rendimiento de la canal fría (RCF), después del sacrificio de los animales, las canales se refrigeraron a $5^{\circ} \mathrm{C}$, por un periodo 24 horas $\mathrm{y}$, luego, se pesaron en una báscula electrónica modelo PB2015 ( $D=100 \mathrm{~g})$. El RCF, se estimó con base en la siguiente fórmula: (PCF/PV*100), donde, PCF correspondió al peso de la canal fría y PV correspondió al peso vivo del animal al momento del sacrificio (Restrepo et al. 2001).

Análisis estadístico. Los resultados correspondientes a la GDP fueron analizados a través de una prueba $\mathrm{t}$ de Student. Los resultados del RCF, se analizaron por medio de la prueba de rango de Wilcoxon. Los datos fueron analizados usando el programa SAS (SAS, 2001).

El análisis estadístico, se realizó mediante el siguiente el modelo estadístico:

$\mathrm{Y}_{i j k}=\mu+\alpha_{\mathrm{i}}+\beta_{\mathrm{j}}+\tau_{\mathrm{k}}+\mathrm{e}_{\mathrm{ijk}}$
En dónde;

$Y_{i j k}=$ Ganancia de peso diaria

$\mu=$ Promedio general

$\alpha_{\mathrm{i}}=$ Efecto de la i-ésima semana (tiempo)

$\beta_{\mathrm{j}}=$ Efecto de la j-ésima repetición

$\tau_{\mathrm{k}}=$ Efecto del k-ésimo tratamiento (suplementación)

$\mathrm{e}_{\mathrm{ijk}}=$ Efecto aleatorio de la varianza

\section{RESULTADOS Y DISCUSIÓN}

La GDP posdestete fue de 29,36 $29,4 \mathrm{~g} / \mathrm{animal}$, en los animales no suplementados y 64,68 8 , $1 \mathrm{~g}$ /animal, en los suplementados. En la figura 1, se puede notar la tendencia de la ganancia de peso promedio por semana, en los dos tratamientos utilizados, evidenciándose que hubo efecto altamente significativo $(\mathrm{P}<0,01)$, entre los tratamientos. La baja ganancia de peso de los animales de este estudio puede estar relacionada con la prevalencia de altas cargas parasitarias y problemas pódales en la época de lluvia.

El aumento en la GDP, por efecto de la suplementación, se ha indicado en muchos estudios, especialmente, cuando se proporciona a animales en épocas críticas (Medina \& Sánchez, 2006; González et al. 2011).

La GDP, del presente estudio, fue inferior $(\mathrm{P}<0,05)$ a la reportada por Martínez et al. (2002), en corderos West African, en Venezuela, quienes encontraron GDP de 133,83 $\pm 0,03 \mathrm{~g} /$ día y $146,46 \pm 0,04 \mathrm{~g} /$ día, en los animales suplementados y $87,40 \pm 0,02 \mathrm{~g} /$ día, en los no suplementados.

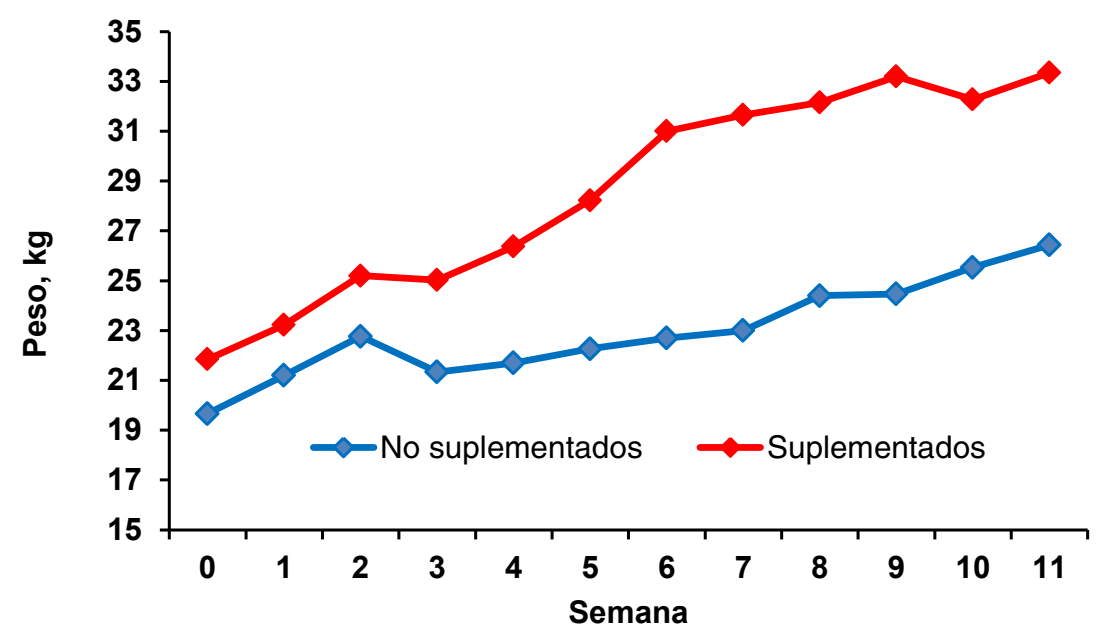

Figura 1. Promedio semanal para la ganancia diaria de peso en ovinos de raza Sudan suplementados y no suplementados. 
Resultados superiores $(\mathrm{P}<0,001)$ fueron reportados por Archimede et al. (2008), en corderos Ovin Martinik, en los grupos suplementados 166 a 203g/día y, en los no suplementados, 134g/día. De igual manera, Duarte \& Pelcastre (2000) hallaron resultados superiores $(P<0,01)$ en la GDP, posdestete en corderos Pelibuey y Pelibuey $\mathrm{x}$ Hampshire, suplementados $226 \pm 36$ g/día y $206 \pm 42$ g/día y no suplementados, $110 \pm 35 \mathrm{~g} /$ día.

Gutiérrez et al. (2005) encontraron resultados superiores $(P>0,05)$, a los del presente estudio, en la GDP posdestete en corderos West African, suplementados 94g/día. González et al. (2002), también hallaron resultados superiores $88 \pm 2 \mathrm{gr} /$ día en la GDP posdestete en corderos Blackbelly, suplementados con un alimento energético-proteico, en el trópico húmedo de México. Mientras que Cadenas et

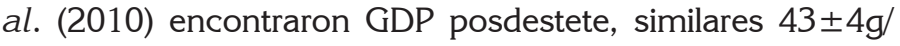
día, a los de este estudio en corderas Pelibuey x Blackbelly, suplementadas con un alimento energético-proteico, en México.
Al observar las medias para cada tratamiento, se destaca la superioridad de la GDP, con el uso de suplementación, debido a que los corderos suplementados obtuvieron una ganancia de peso mayor que los no suplementados, durante el periodo experimental (Figura 1). Estos resultados sugieren que la GDP pudo estar relacionada con el mejor aporte de energía y de proteína del suplemento, sugiriendo un efecto estimulador del consumo por parte de la combinación maízsoya, al mejorarse la relación energía-proteína, favoreciendo la actividad microbiana ruminal, incrementando la digestibilidad y la velocidad de pasaje de la digesta y, por ende, el consumo (Alliston, 1985); sin embargo, no se midió el consumo de forraje durante el pastoreo de los corderos, razón, por la cual, no se pudo separar el efecto del suplemento y forraje con relación a la ganancia de peso.

El RCF, del presente estudio, fue 35,05 $\pm 5,5 \%$ en los animales no suplementados y $40,46 \pm 2,04 \%$, en los suplementados; no se encontró diferencia estadística $(P>0,05)$, entre los tratamientos (Tabla 1).

Tabla 1. Rendimiento de la canal fría de corderos de raza Sudan suplementados y no suplementados.

\begin{tabular}{|c|c|c|c|}
\hline TRATAMIENTOS & $\mathbf{n}$ & GDP (gr) & RCF (\%) \\
\hline No suplementados & 3 & $29,36 \pm 9,4 \mathrm{a}$ & $35,05 \pm 5,55 \mathrm{a}$ \\
\hline Suplementados & 4 & $64,68 \pm 8,1 \mathrm{~b}$ & $40,46 \pm 2,04 \mathrm{a}$ \\
\hline
\end{tabular}

Valores medios con diferente letra en la misma columna, difieren estadísticamente $(P \leq 0,05)$.

El RCF, del presente estudio, fue similar a lo reportado por Archimede et al. (2008), en corderos Ovin Martinik; estos autores reportaron rendimientos de la canal del 39\%, en los corderos no suplementados y del 41 a $46 \%$, en los suplementados.

Resultados superiores fueron reportados en corderos de raza Omani, en los grupos no suplementados, $47,5 \pm 0,8 \%$ y, suplementados, 44,5 a $47,7 \pm 0,8 \%$, respectivamente (Mahgoub et al. 2000). De igual manera, Burke et al. (2003) y Gutiérrez et al. (2005) encontraron RCF, superiores 49 a $53,7 \pm 3,11 \%$ y $43,96 \pm 0,63$ a $44,49 \pm 0,66 \%$, respectivamente, en corderos mestizos.

Los resultados, del presente estudio, indican que la suplementación no tuvo efecto significativo sobre el RCF de los corderos, aunque estadísticamente las diferencias estuvieron cercanas a valor de significancia $(P=0,05)$, por lo que se recomienda realizar otro experimento en época seca y con mayor número de animales. Esta baja respuesta a la suplementación difiere a lo reportado por Burke et al. (2003) y Gutiérrez et al. (2005), quizás debido al bajo potencial genético de los animales utilizados, ya que, en este estudio, se trabajó con animales de raza Sudan, a la cual, no se le ha realizado mejoramiento genético, mientras que los otros autores trabajaron con razas mejoradas.

Agradecimientos. Los autores agradecen a la Universidad de Córdoba, por facilitar los animales para la realización de este estudio. Conflicto de intereses: El manuscrito fue preparado y revisado con la participación de todos los autores, quienes declaramos que o existe ningún conflicto de intereses, que ponga en riesgo la validez de los resultados presentados.

\section{BIBLIOGRAFÍA}

1. ALLISTON, C.D. 1985. Factors affecting forage intake by range rumiants: a review. J. Range Manage. 38:305. 
2. ASSOCIATION OF OFFICIAL ANALYSIS CHEMISTRY -AOAC-. 2002. Official Method 2002.04. AmylaseTreated Neutral detergent Fiber in Feeds. AOAC Official Methods of Analysis Association of Official Analytical Chemists. (USA).

3. ARCHIMEDE, H.; PELLONDE, P.; DESPOIS, P.; ETIENNE, T.; ALEXANDRE, G. 2008. Growth performances and carcass traits of Ovin Martinik lambs fed various ratios of tropical forage to concentrate under intensive conditions. Small Rumin. Res. 75:162-170.

4. BURKE, J.M.; APPLE, J.K.; ROBERTS, W.; BOGER, C.B.; KEGLEY, E.B. 2003. Effect of breed-type on performance and carcass traits of intensively managed hair sheep. Meat Sci. 63:309-315.

5. CADENAS, J.A.; OLIVA, J.; HINOJOSA, J.A.; TORRES, G. 2010. Suplementación postdestete de corderas pelibuey $\mathrm{x}$ blackbelly en pastoreo en el trópico húmedo. Arch. Zootec. 59(226):303-306.

6. DUARTE, F.; PELCASTRE, A. 2000. Efecto de la suplementación predestete a corderos en condiciones tropicales. Livest. Res. Rural. Dev. 12(3).

7. GONZÁleZ, G.R.; TORRES, G.; CASTILLO, M.A. 2002. Crecimiento de corderos Blackbelly entre el nacimiento y el peso final en el trópico húmedo de México. Vet. Méx. 33:443-453.

8. GONZÁlEZ, R.; TORRES, G.; ARECE, J. 2011. Ganancia de peso de ovinos alimentados con pasto Taiwán (Pennisetum purpureum) suplementados con diversas fuentes de proteína. Avances en Investigación Agropecuaria. 15(3):3-20.

9. GUTIÉRREZ, J.; RUBIO, M.S.; MÉNDEZ, R.D. 2005. Effects of crossbreeding Mexican Pelibuey sheep with Rambouillet and Suffolk on carcass traits. Meat. Sci. 70:1-5.

10. ICONTEC. 1999. Alimentos para animales, determinación del contenido de nitrógeno y cálculo de proteína cruda. Método Kjeldahl-NTC 4657, Publicaciones ICONTE. (Colombia).
11. ICONTEC. 2006. Alimentos para animales, determinación de ceniza cruda-NTC 4648, Publicaciones ICONTEC, (Colombia).

12. IZAGUIRRE, F.; MARTÍNEZ, T.J.J.; JIMÉNEZ, F.J.G.O.; POSADA, C.S.; GARCÍA, C.C.G.; MARTÍNEZ, P.G. 2011. Respuesta reproductiva y productiva de borregas pelibuey a la suplementación con hojas de Caulote (Guazuma ulmifolia), Guaje (Leucaena leucocephala) y Yaite (Gliricidia sepium) en condiciones de trópico húmedo. Livest. Res. Rural. Dev. 23(10).

13. MAHGOUB, O.; LU, C.D.; EARLY, R.J. 2000. Effects of dietary energy density on.feed intake, body weight gain and carcass chemical composition of Omani growing lambs. Small. Rumin. Res. 37:35-42.

14. MARTÍNEZ, M.; BRAVO, J.O.; BETANCOURT, M.; BRACHO, I.; QUINTANA, H. 2002. Influencia de la suplementación proteica sobre el crecimiento de corderos post destete. Zootecnia. Trop. 20(3):307-317.

15. MEDINA, R.; SÁNCHEZ, A. 2006. Efecto de la suplementación con follaje de Leucaena leucocephala sobre la ganancia de peso de ovinos desparasitados y no desparasitados contra estrongílidos digestivos. Zootecnia. Trop. 24(1):55-68.

16. MINISTERIO DE AGRICULTURA Y DESARROLLO RURAL -MADR-. 2006. La cadena ovinos y caprinos en Colombia. Documento de trabajo $\mathrm{N}^{\circ} 125$. Disponible desde Internet en: http://201.234.78.28:8080/ dspace/bitstream/123456789/907/1/2007861135 7caracterizacion_ovinosycaprinos.pdf (con acceso 15/06/14).

17. RESTREPO, D.; ARANGO, C.; AMEZQUITA, A.; RESTREPO, R. 2001. Industria de la carne. U. Nacional de Colombia. (Colombia). p.27-32.

18. STATISTICAL ANALYSIS SYSTEMS -SAS-. 2001. User's guide (version 9.1) Cary (USA). Institute SAS/STAT.

Recibido: Julio 25 de 2014

Aceptado: Febrero 21 de 2015

\section{Cómo citar:}

Maza Angulo, L.; Bustamante Yanez, M.; Simanca Sotelo, J.C.; Ruiz Ortiz, M.; Montaño Flórez, G.; Vergara Garay, O. 2015. Efecto de la suplementación sobre la ganancia de peso y rendimiento en canal de corderos Sudan. Rev. U.D.C.A Act. \& Div. Cient. 18(1): 283-286. 